\title{
The internet is leading the world towards forms of totalitarianism: How to fix the problem
}

It is difficult to imagine intelligence studies as separate from information technology as we enter the third decade of the 21 st century. The current issue of JISIB bears witness to this integration with a strong focus on big data applications.

Hardly anyone today would or could do without the internet, but the project that started with US government financing in the 1960s, with packet switching, and in the 1970s with ARPANET and saw commercial light in the 1990s is helping countries turn into totalitarian systems where totalitarianism is defined by a high degree of control over public and private life.

Public life is influenced by hacking, troll factories, fake news/propaganda, and interference in elections. Private life is influenced by massive surveillance. To borrow the title of the book by Zuboff (2019) we now live in "the age of surveillance capitalism". Business intelligence systems lie at the heart of this transformation, but so do artificial intelligence and robotics. And the trend is global.

In the West the suppressors are mostly private monopolies (e.g. Google, Facebook), while in the East it is primarily the government that is snooping (e.g. China's Social Credit System). Face recognition is likely to become as popular in the West as it is in the East. It is also easily forgotten that no city was better surveilled than London, which started to build its CCTV technology in the 1960s. The system is now being updated with facial recognition, just like the one we are criticizing the Chinese for having. Some forms of surveillance may also lead to great advances in our societies, like access to government forms and statements electronically and a non-anonymous Central Bank Digital Currency (CBDC), which promises to reduce corruption and tax fraud, and could be used for easy distribution of universal basic income (UBI) . Fintech promises to be highly disruptive.

We are moving into an Orwellian world of surveillance more or less voluntarily, often applauding it. "I have nothing to hide" the young man says, but then he later becomes a minister and starts to worry about the traces he has left on keyboards. The Five Eyes intelligence alliance, or any other major service, can pull out extensive analyses of behavior and personality on most of us now as we continue to exchange our personal data for access to searches and social media, but also subscription-based services. Most Chinese think that the social credit system is a good thing. This is for much of the same reason: they believe it will not be used against them and think that they will do well. We all tend to be overoptimistic about our abilities and opportunities. It's not before we fail that the full implications of the system are felt: lack of access, credit, housing, and no more preferential treatments. The result threatens to worsen the lack of social mobility and increase the growing conflict between the super-rich and those hundreds of millions who risk slipping from the middle class to being counted among the poor, many of whom live in the Western world.

The truth is another essential part of our civilization that we are now tampering with. On the internet, few users can tell facts from lies, but we think we can. Most of those who grew up only with the internet never really learned how to think critically. The old library of physical books was the best guarantee that lessons learned from history would be transferred to future generations without anyone mingling. For that same reason, books were also seen as real threats to tyrants and have been censured and burned. The last time that happened in the West on a large scale was in Nazi Germany, but it is happening again now in subtler forms as Amazon and other giants act as arbiter and refuse books with certain content based on value judgements. A world which relies all too much on the internet should recall that the information there can be switched off in a second. Old books are often not even accessible, having been exchanged for online solutions. The situation in the brave new social sciences is much the same, everyone is running after the latest articles without ever questioning if the same ideas have been published before (difficult to know now). Thus, much academic literature suffers, becoming a tedious process of repetitions under new brands. In a society where everyone is a writer, no one really reads or has much of importance to say at the end. 
How do we solve these problems? Step one on the internet is serious encryption as to make data private. Step two is to give all personal data back to the users, that is, to take it away from the private companies and then indirectly away from the security services. That will eliminate the "free" business model and lead to more subscription-based products instead. Step three is to break up the monopolies, and before that to tax them properly. Step four is to return to books that have stood the test of time (real peer-reviewed) whether online or offline. (The learning process is probably only half as good on the screen). We need to go from a culture of skimming data back to reading and discussing it. Technology and management practices should be a part of that solution. Otherwise it looks like we will continue down the road that leads to totalitarianism. The internet right now is making shopping easier, but most people are becoming less aware of realities, less smart, less critical. Only a small part of the population is able to use it to their advantage for understanding the world around them. It would be great to see more articles develop ideas and products for how we as societies can go in this direction.

When looking at the articles in this issue we are reminded that intelligence studies is no longer an Anglo-Saxon body of literature but has become truly international.

The first article entitled "Interpreting, analyzing and distributing information: a big data framework for competitive intelligence" is by Erlaine Binotto et al. It presents a big data intelligence framework.

The second article entitled "Competitive intelligence and absorptive capacity for enhancing innovation performance of SMEs" by Abdeslam Hassani and Elaine Mosconi suggests a way in which competitive intelligence enhances innovation performance for SMEs.

The third article entitled "A framework for big data integration within the strategic management process based on a balanced scorecards methodology" by Mouhib Alnoukari shows how to integrate big data into the strategic management process using a balanced scorecards methodology.

The fourth article entitled "Competitive intelligence approach for developing an e-tourism strategy post COVID-19" by Franky Tulungen et al. shows a strategy for how to boost tourism after the COVID19 pandemic by developing e-tourism based on a competitive intelligence approach.

The fifth article entitled "La veille stratégique entre l'efficacité décisionnelle et l'optimisation de la gouvernance: etude restreinte dans les organismes publics tunisiens" by Mostapha Tayeb Ben Amor and Fatma Chichti (in French, abstract in English) suggests an intelligence framework for the public sector. The study is based on interviews with public sector organizations in Tunisia.

The last article entitled "Integrating science and technology metrics into a competitive technology intelligence methodology" by Marisela Rodriguez-Salvador and Pedro F. Castillo-Valdez presents a new framework for competitive technology intelligence (CTI) providing a broader scope to science and technology metrics where quantitative tools such as patentometrics and scientometrics are used.

As always, we would above all like to thank the authors for their contributions to this issue of JISIB. Thanks to Dr. Allison Perrigo for reviewing English grammar and helping with layout design for all articles. I wish I could say that the COVID-19 pandemic is soon over, but unfortunately it still has a grip on our societies.

On behalf of the Editorial Board,

Sincerely Yours,

$$
\text { Kuñn 1. Nin }
$$

Prof. Dr. Klaus Solberg Søilen

Halmstad University, Sweden

Editor-in-chief

Zuboff, S. (2019). The age of surveillance capitalism: The fight for a human future at the new frontier of power: Barack Obama's books of 2019. Profile books. 